\title{
Effect of Vitamin C and Atorvastatin on Male Fertility in Rats Subjected to Forced Swimming Stress
}

\author{
Balaji Ommurugan ${ }^{1}$, Vanishree Rao ${ }^{2}$, Amod Tilak $^{3}$, Kurady L Bairy ${ }^{4}$
}

\begin{abstract}
Introduction: An imbalance between the oxidative system and the antioxidant defense system leads to stress. So the aim of this study is to investigate the effect of vitamin $\mathrm{C}$ and atorvastatin on fertility in male rats subjected to experimental stress using the forced swimming stress model.

Materials and methods:Thirty adult male albino rats weighing 200-250 $\mathrm{g}$ and aged 10-12 weeks were used. Institutional Animal Ethics Committee approval was obtained. Rats were exposed to forced swimming stress daily and drugs were orally administered 30 minutes prior to the stress for a total of 15 days once daily. Group I: the nonstress group with distilled water (negative control); group II: positive control (only stress) plus distilled water; group III: stress + vitamin C (20 mg/kg/day); group IV: stress + atorvastatin ( $2 \mathrm{mg} / \mathrm{kg} /$ day); group V: stress + vitamin C (20 mg/kg/day) + atorvastatin ( $2 \mathrm{mg} / \mathrm{kg} /$ day). On the 16th day, the effect of drugs in stressed rats on body weight, testicular and epididymis weight, testicular index, sperm count, and motility was assessed.

Results: In stressed rats, sperm count, motility, testicular weight, epididymis weight, and testicular index were significantly reduced when compared with the control group. Groups III, IV, and V showed a significant increase $(p<0.001)$ in all five parameters when compared with the stress group.

Conclusion: The results suggest that vitamin $C$ and atorvastatin significantly protected the harmful effect of stress on sperms.

Clinical significance: The pleiotropic antioxidant effect of statins is proven by this study and clinical trials can be done to evaluate the role of atorvastatin in treating male infertility.

Keywords: Antioxidants, Atorvastatin, Forced, Lipid peroxidation, Pleiotropy.

International Journal of Infertility and Fetal Medicine (2018): 10.5005/.jp-journals-10016-1171
\end{abstract}

\section{INTRODUCTION}

In a social set-up, like India, with greater emphasis being thrown on childbearing, infertility results in a psychological as well as an economic burden with medical implications. According to the World Health Organization, infertility is defined as a failure to achieve pregnancy after 12 months of regular unprotected sexual intercourse. ${ }^{1}$ About 60-80 million couples suffer from infertility per recent estimates by the World Health Organization. ${ }^{2}$ It almost affects $8-12 \%$ of couples worldwide with regional variations. ${ }^{3,4}$ Per recent studies, a prevalence of infertility in India ranges between $3.9 \%$ and $16.8 \%$ with $40 \%$ of those infertility cases having a male factor as the main etiology. ${ }^{2,5}$ Male infertility is defined as an inability of the male partner to result in pregnancy in a fertile female factor. Male infertility usually results in alterations in sperm counts as well as sperm characteristics which includes motility, morphology, and viability. ${ }^{6}$ Globally, the incidence of male infertility is said to be around $40-50 \%$ affecting $7 \%$ of men. ${ }^{7-10}$

An imbalance between the oxidative system and the antioxidant defense system leads to stress. The homeostasis of the body is put to various external and internal demands, which involves the proper mobilization of physiological and psychological resources to deal them. ${ }^{11}$ Oxidative stress is found to be one of the leading causes of infertility in males. ${ }^{12}$ Normally, a balance exists between reactive oxygen species and antioxidant scavenging activities in male reproductive organs. ${ }^{13}$ Several studies have examined the association of stress and sexual behavior in male rats. ${ }^{12,14}$

Lipid peroxidation is one of the main events induced by oxidative stress and is mainly produced by a cascade of free radicals. Spermatogenesis, being a process of high cell division, involves
${ }^{1-3}$ Department of Pharmacology, Kasturba Medical College, Manipal, Karnataka, India

${ }^{4}$ Department of Pharmacology, RAK College of Medical Sciences, RAK Medical and Health Science University, United Arab Emirates

Corresponding Author: Balaji Ommurugan, Department of Pharmacology, Kasturba Medical College, Manipal, India, Phone: +91 9500140439, e-mail: puntermmc@gmail.com

How to cite this article: Ommurugan B, Rao V, et al. Effect of Vitamin C and Atorvastatin on Male Fertility in Rats Subjected to Forced Swimming Stress. Int J Infertil Fetal Med 2018;9(3):27-31.

Source of support: Nil

Conflict of interest: None

increased mitochondrial oxygen consumption by the germinal epithelium of testis. Since the tissue oxygen concentration of testis is very low, there is an intense competition for the vital elements of the testis and this makes the testis more protective against oxidative damage. ${ }^{15}$ Despite this protective microenvironment, testicular membranes are rich in polyunsaturated fatty acids and, thus, susceptible to peroxidation injury. ${ }^{15}$ Increased lipid peroxidation in the testis contributes to the suggested vulnerability of this organ to oxidative stress. So to protect this, the testis has an antioxidant defense system characterized by various enzymes like nicotinamide adenine dinucleotide phosphate (reduced form with the $\mathrm{H}$ ion) [NADPH] reductase, glutathione peroxidase, superoxide dismutase, and catalase. Apart from these enzymes, there are various small molecule factors protecting the testis from oxidative damage including zinc, vitamin C, vitamin E, melatonin, and cytochrome $\mathrm{C}^{15}$ 
Ascorbic acid is present in the testis helping as an important antioxidant defense. ${ }^{16}$

Dehydroascorbate reductase is abundant in the testis and this helps in maintaining the vitamin $C$ in the reduced state in the testis. ${ }^{17}$ Vitamin $C$ supplementation helps in improving sperm counts. Vitamin $C$ has shown to increase sperm parameters in male rats subjected to stress. ${ }^{18}$ Statins apart from being a lipid-lowering agent have many more pleiotropic effects. The role of statins as an antioxidant has been recently studied widely and it has been found that statins are effective antioxidant because of its ability to inhibit distinctive oxidation pathways and also its role in the inhibition of myeloperoxidative enzymes and NO levels. ${ }^{19}$

So the purpose of this study is to evaluate the role of vitamin C and atorvastatin in male fertility in rats subjected to stress.

\section{Materials and Methods}

The study was commenced after getting approval from the Institutional Animal Ethics Committee.

\section{Chemicals/Drugs}

Vitamin C and atorvastatin were procured from Kasturba Medical Hospital Pharmacy. Phosphate-buffered saline and other surgical items were obtained from Pharmacology Laboratory, Kasturba Medical College, Manipal.

\section{Animals}

Male albino rats of Wistar strain weighing 200-250 $\mathrm{g}$ and 10-12 weeks were used for this study. The animals were housed under standard condition, 12:12 light-dark cycle, 50\% humidity, and $28^{\circ} \mathrm{C}$ temperature and provided with standard food granules and water ad libitum.

\section{Forced Swimming Stress Model}

The forced swimming stress model designed by Porsolt et al. ${ }^{20}$ was used in the study with modifications based on Nayanatara et al. ${ }^{21}$ Each rat was subjected to swimming in a glass tank measuring a width of $40 \mathrm{~cm}$, a depth of $60 \mathrm{~cm}$, and a length of $100 \mathrm{~cm}$. Rats were allowed to swim inside the tank with a water level of $35 \mathrm{~cm}$, so that rats cannot go and rest its tail on the tank. The cutoff point for exhaustion was assessed by noting the time and if the rats are unable to come up and breathe within 7 seconds, the rats were considered exhausted and they were removed from the tank.

\section{Experimental Design}

Thirty adult male rats with an average body weight of $200-250 \mathrm{~g}$ were used for this study. The rats were randomly divided into five groups of six rats each. The rats were dosed 30 minutes before swimming stress daily for 15 days. All drugs were given orally. A dose of atorvastatin was taken as $2 \mathrm{mg} / \mathrm{kg}$ and vitamin $C$ as $20 \mathrm{mg} / \mathrm{kg}$ per previous studies in literature. ${ }^{18}$ The rats are divided into five groups as follows:

Group I: normal control animal received distilled water.

Group II: positive control (only stress) plus distilled water.

Group III: stress + vitamin C ( $20 \mathrm{mg} / \mathrm{kg} /$ day).

Group IV: stress + atorvastatin ( $2 \mathrm{mg} / \mathrm{kg} /$ day).

Group V: stress + vitamin C (20 mg/kg/day) + atorvastatin ( $2 \mathrm{mg} / \mathrm{kg} /$ day).

At the end of the study period, all rats were sacrificed by cervical dislocation on the 16th day and rats were dissected and reproductive organs were removed. The weight of the testis and the epididymis were measured. Sperm counts, motility, viability, and live and dead sperm ratio (LDR) were estimated.

\section{Methods \\ Body Weight}

Before the start of the study and the end of the experimental period, the body weight of each individual rat was measured and recorded.

\section{Reproductive Organ Weights}

Reproductive organs: testes and epididymis were dissected out and were weighed.

\section{Testicular Index}

The testicular index is calculated by dividing the left testis weight by the body weight and then multiplied by 100 .

\section{Sperm Analysis \\ Sperm Count}

The epididymis was minced in $1 \mathrm{~mL}$ of phosphate buffer saline (PBS) and the suspension was filtered through a mesh. An aliquot from the suspension (up to 0.5 ) is taken in a hemocytometer and was mixed with phosphate-buffered saline in 1:10 dilution. The suspension is then charged into a Neubauer's counting chamber. The total sperm count in eight squares (except the central erythrocyte area) of $1 \mathrm{~mm}^{2}$ each was determined and multiplied by $5 \times 10^{4}$ to express the number of spermatozoa/epididymis. ${ }^{22}$

\section{Sperm Motility}

It was done by the method described by Saalu et al. ${ }^{23}$ The epididymis was macerated and mixed with $1 \mathrm{~mL}$ PBS. An aliquot of this solution was taken on the slide and the percentage of motility was evaluated microscopically at a magnification of $400 \times$. Three different fields were chosen from each sample. The mean of the three estimations was used as the final motility score.

\section{Live and Dead Sperms (Viability)}

A drop of the epidydimal content of each rat was mixed with an equal drop of $1 \%$ eosin stain as described by Metwally et al. ${ }^{24}$ Thinfilm slides were made and allowed to dry quickly. Viable sperm remains colorless. One hundred sperm cells per rat were used for scoring and determining the viability percent.

\section{Results}

\section{Effect on Body Weight}

All baseline values were comparable, and there was no statistically significant difference $(p>0.05)$ among them as given in Table 1. There was a significant reduction in body weights of the stress group (196.67 $\pm 6.79 ; p<0.001)$, the vitamin C group (214.17 \pm $8.11 ; p<0.01)$, the atorvastatin group $(200.83 \pm 1.54 ; p<0.001)$, and the atorvastatin plus vitamin C group $(208.34 \pm 7.49 ; p<0.01)$ when compared with the control group $(247.50 \pm 4.43)$ at the end of 15th day. The reduction in body weight of treatment groups was not significant $(p>0.05)$ when compared with the stress group. The intragroup analysis showed a significant difference in the bodyweight of all groups (Table 1). 
Table 1: Body weight and reproductive organ weight

\begin{tabular}{llllll}
\hline Group $n=6$ & Testis weight $(\mathrm{mg})$ & Epididymis weight $(\mathrm{mg})$ & Testicular index\% & $\begin{array}{l}\text { Body weight on } \\
\text { day } 1(\mathrm{~g})\end{array}$ & $\begin{array}{l}\text { Body weight on } \\
\text { day 30 }(\mathrm{g})\end{array}$ \\
\hline Control & $4.45 \pm 0.04$ & $1.33 \pm 0.48$ & $0.90 \pm 0.04$ & $240.83 \pm 3.27$ & $247.50 \pm 4.43$ \\
Stress group & $2.38 \pm 0.07^{\#}$ & $0.28 \pm 0.01^{\#}$ & $0.61 \pm 0.05^{\#}$ & $229.17 \pm 8.41$ & $196.67 \pm 6.79^{\#}$ \\
Vitamin C (test) & $3.43 \pm 0.07^{\# *}$ & $0.66 \pm 0.04^{\# *}$ & $0.81 \pm 0.11^{\# *}$ & $227.50 \pm 6.16$ & $214.17 \pm 8.11^{\#}$ \\
Atorvastatin (test) & $3.28 \pm 0.09^{\# *}$ & $0.52 \pm 0.02^{\# *}$ & $0.82 \pm 0.06^{\# *}$ & $210.83 \pm 2.71$ & $200.83 \pm 1.54^{\#}$ \\
Vitamin C + atorvastatin (test) & $3.46 \pm 0.07^{\# *}$ & $0.56 \pm 0.04^{\# *}$ & $0.83 \pm 0.07^{\# *}$ & $221.67 \pm 7.92$ & $208.34 \pm 7.49^{\#}$ \\
\hline
\end{tabular}

${ }^{*} p<0.001$ : stress group vs all other groups

$\# p<0.001$ : test groups vs control group. Mean \pm SEM was taken and intergroup analysis done with one-way ANOVA followed by Tukey's post hoc test with $p<0.05$ as significance

\section{Effect on Testicular Weight}

There was a significant reduction in the weight of the testis in the stress group $(2.38 \pm 0.07 ; p<0.001)$, vitamin $C$ group $(3.43 \pm 0.07 ; p<0.001)$, atorvastatin group $(3.28 \pm 0.09 ; p<0.001)$, and atorvastatin plus vitamin C group $(3.46 \pm 0.07 ; p<0.001)$ when compared with the control group $(4.45 \pm 0.04)$. The stress group showed a significant $(p<0.001)$ reduction in the weight of the testis when compared with all the three treatment groups. There was no significant difference with respect to testicular weight reduction among the treatment groups as in Table 1.

\section{Effect on Epididymis Weight}

There was a significant reduction in the weight of the epididymis in the stress group $(0.28 \pm 0.01 ; p<0.001)$, vitamin $C$ group $(0.66 \pm 0.04 ; p<0.001)$, atorvastatin group $(0.52 \pm 0.02 ; p<0.001)$, and atorvastatin plus vitamin C group $(0.56 \pm 0.04 ; p<0.001)$ when compared with the control group $(1.33 \pm 0.48)$. The stress group showed a significant $(p<0.001)$ reduction in the weight of the epididymis when compared with all the three treatment groups. There was no significant $(p>0.05)$ difference with respect to testicular weight reduction among the treatment groups as in Table 1.

\section{Effect on Testicular Index}

There was a significant reduction in the testicular index in the stress group $(0.61 \pm 0.05 ; p<0.001)$, vitamin C group $(0.81 \pm 0.11 ; p<0.01)$, atorvastatin group $(0.82 \pm 0.06 ; p<0.001)$, and atorvastatin plus vitamin C group $(0.83 \pm 0.07 ; p<0.01)$ when compared with the control group $(0.90 \pm 0.04)$. The stress group showed a significant $(p<0.001)$ reduction in the testicular index when compared with all the three treatment groups. There was no significant $(p>0.05)$ difference with respect to the testicular index among the treatment groups as given in Table 1 and Fig. 1.

\section{Effect on Sperm Counts}

There was a significant reduction in sperm counts in the stress group (1656.67 $\pm 37.74 ; p<0.001)$, vitamin C group $(2775.00 \pm 52.31$;

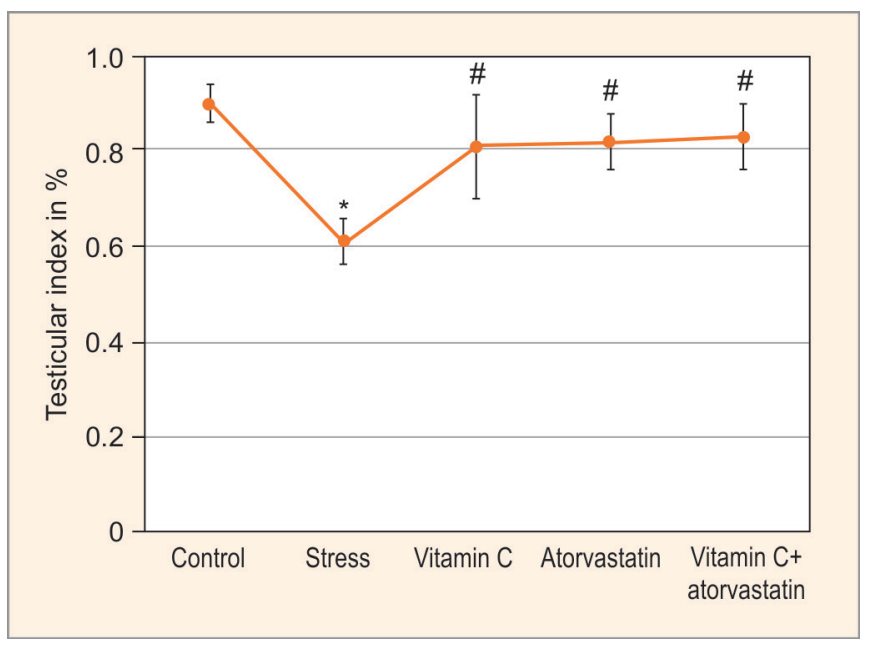

Fig. 1:Testicular index measurement. ${ }^{*} p<0.001$ : stress group vs all other groups, ${ }^{\#} p<0.001$ : test groups vs control group. Mean \pm SEM was taken and intergroup analysis done with one-way ANOVA followed by Tukey's post hoc test with $p<0.05$ as significance

$p<0.001)$, atorvastatin group $(2841.67 \pm 30.10 ; p<0.001)$, and atorvastatin plus vitamin C group $(2675.83 \pm 54.03 ; p<0.001)$ when compared with the control group (3366.67 \pm 105.41$)$. All the three-treatment groups showed a significant $(p<0.001)$ improvement in sperm counts when compared with all the stress groups. There was no significant $(p>0.05)$ difference with respect to improvement in sperm counts among the treatment groups as given in Table 2 and Fig. 2.

\section{Effect on Sperm Motility}

There was a significant reduction in sperm motility in the stress group (34.92 $\pm 0.25 ; p<0.001)$, vitamin C group $(59.09 \pm 0.27$; $p<0.001)$, atorvastatin group (62.91 $\pm 0.27 ; p<0.001)$, and atorvastatin plus vitamin $C$ group $(58.04 \pm 0.12 ; p<0.001)$ when

Table 2: Sperm parameter analysis

\begin{tabular}{lllcc}
\hline Group & Sperm count $\times 10^{4}$ cells & Sperm motility\% & Sperm viability\% & Sperm LDR \\
\hline Control & $3366.67 \pm 105.41$ & $65.51 \pm 0.32$ & $80.0 \pm 1.39$ & $4.10 \pm 0.36$ \\
Stress group & $1656.67 \pm 37.74^{\#}$ & $34.92 \pm 0.25^{\#}$ & $55.0 \pm 2.16^{\#}$ & $1.22 \pm 0.01^{\#}$ \\
Vitamin C (test) & $2775.00 \pm 52.31^{\#}$ & $59.09 \pm 0.27^{\# *}$ & $74.66 \pm 1.39^{\# *}$ & $2.70 \pm 0.30^{\# *}$ \\
Atorvastatin (test) & $2841.67 \pm 30.10^{\#}$ & $62.91 \pm 0.27^{\# *}$ & $77.0 \pm 1.96^{\# *}$ & $3.34 \pm 0.36^{\# *}$ \\
Vitamin C + atorvastatin (test) & $2675.83 \pm 54.03^{\#}$ & $58.04 \pm 0.12^{\# *}$ & $69.83 \pm 2.36^{\# *}$ & $2.22 \pm 0.11^{\# *}$ \\
\hline
\end{tabular}

${ }^{*} p<0.001$ : stress group vs all other groups

${ }^{\#} p<0.001$ : test groups vs control group. Mean \pm SEM was taken and intergroup analysis done with one-way ANOVA followed by Tukey's post hoc test with $p<0.05$ as significance 


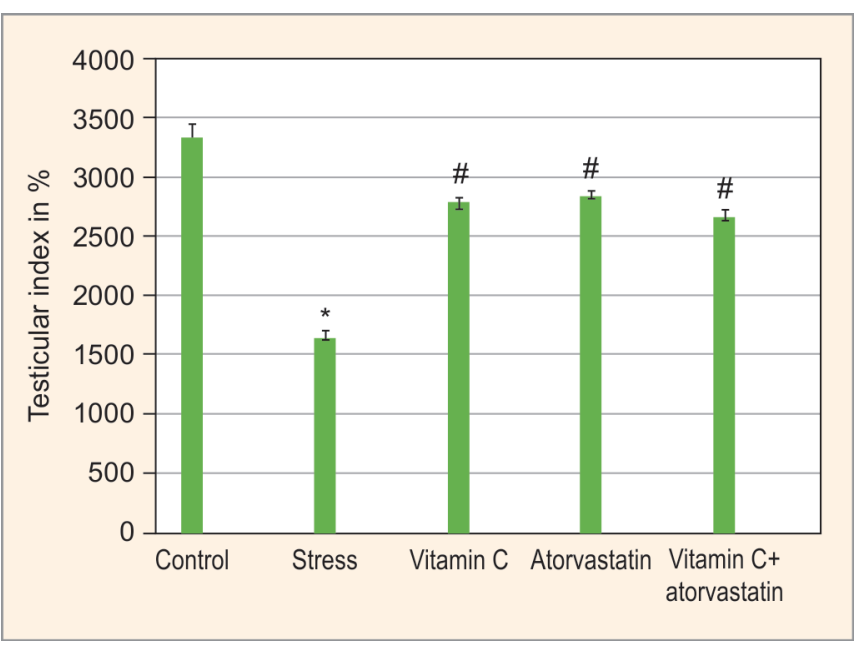

Fig. 2: Sperm counts analysis. ${ }^{*} p<0.001$ : stress group vs all other groups, ${ }^{\#} p<0.001$ : test groups vs control group. Mean \pm SEM was taken and intergroup analysis done with one-way ANOVA followed by Tukey's post hoc test with $p<0.05$ as significance

compared with the control group $(65.51 \pm 0.32)$. All the threetreatment groups showed a significant $(p<0.001)$ improvement in sperm motility when compared with the stress group. There was no significant $(p>0.05)$ difference with respect to sperm motility among the treatment groups as given in Table 2.

\section{Effect on Sperm Viability}

There was a significant reduction in the viability of sperms in the stress group $(55.0 \pm 2.16 ; p<0.001)$, vitamin $C$ group $(74.66 \pm$ 1.39; $p<0.001)$, atorvastatin group (77.0 $\pm 1.96 ; p<0.001)$, and atorvastatin plus vitamin C group $(69.83 \pm 2.36 ; p<0.001)$ when compared with the control group $(80.0 \pm 1.39)$. All the threetreatment groups showed a significant $(p<0.001)$ increase in the sperm viability when compared with the stress group. There was no significant $(p>0.05)$ difference with respect to sperm viability among the treatment groups as given in Table 2 .

\section{Effect on LDR}

There was a significant reduction in LDR in the stress group $(1.22 \pm$ $0.01 ; p<0.001)$, vitamin C group ( $2.70 \pm 0.30 ; p<0.001)$, atorvastatin group $(3.34 \pm 0.36 ; p<0.001)$, and atorvastatin plus vitamin C group $(2.22 \pm 0.11 ; p<0.001)$ when compared with the control group $(4.10 \pm 0.36)$. All the three-treatment groups showed a significant $(p<0.001)$ increase in LDR when compared with the stress group. There was no significant $(p>0.05)$ difference with respect to LDR among the treatment groups as given in Table 2.

\section{Discussion}

Summarizing the results, stress group, vitamin $C$ group, atorvastatin group, and vitamin C plus atorvastatin group showed a significant reduction in total body weight, reduction of reproductive organ weights, and also a significant reduction in sperm parameters when compared with the control group. There was no difference in body weight among the treatment groups in comparison with the stress-only group. There was a significant recovery in weights of reproductive organs among treatment groups when compared with the stress group without any treatment. With respect to sperm parameters, all groups showed a significant decrease compared to the control group.
The treatment groups showed a significant improvement in sperm parameters in comparison with the stress-only group. There was no significant difference noted between treatment groups with respect to all the parameters.

Stress is implicated as one of the leading causes of male infertility. We found that there was a significant reduction in body weight, reproductive organ weight, as well as in sperm parameters in rats subjected to swimming stress. All groups subjected to the forced swim test showed a decrease in body weight compared to the control group. Similar results are observed by earlier workers. ${ }^{18}$ The possible mechanism that can be attributed to this reduction in body weight among rats subjected to stress is reduced food intake as well as release of corticotrophin-releasing hormone leading to loss of appetite. ${ }^{25,26}$

In this study, the control group without stress showed a significant difference $(p<0.001)$ in the weight of the testis, the testicular index as well as the epididymis weight when compared to all other groups subjected to stress. Vitamin $C$, atorvastatin, and their combination groups showed a significant $(p<0.001)$ recovery in the testicular, the testicular index, and the epididymis weight when compared to the stress group without treatment. Vitamin C, atorvastatin, and their combination group were able to show recovery in weights of reproductive organs and testicular index but not fully reverse the changes caused by stress. The effect produced by vitamin $C$ was in accordance with previous studies done. ${ }^{18}$ A possible mechanism for this reduction in weights of reproductive organs among stressed animals can be due to the influence of stress on sex hormone production. ${ }^{18}$

All groups had a significant $(p<0.001)$ decrease of all sperm parameters when compared to normal control rats. Vitamin C, atorvastatin, and their combination group showed a significant $(p<0.001)$ improvement in the sperm parameters when compared to the stress group without treatment. There was no significant difference $(p>0.05)$ among vitamin $C$, atorvastatin, and their combination groups with respect to sperm parameters.

So, in our study, we observed both vitamin $C$ and atorvastatin groups helped in improving the sperm parameters and helped in reversing the fertility changes caused by swimming stress but not fully back to normal. The effects of vitamin C observed in our study are similar to the results in the previous study where $20 \mathrm{mg} / \mathrm{kg}$ of vitamin $C$ used as an intermediate dose helped in improving the sperm parameters and reversing the fertility changes produced by stress. ${ }^{18}$ Vitamin $C$ was via its antioxidant effect in the testicular tissue helps in protecting spermatogenesis and prevents oxidative damage. ${ }^{27}$

An effect produced by atorvastatin in our study is similar to results produced in a study in rats where Simvastatin was used to reverse fertility changes produced in hypercholesterolemic rats. ${ }^{28}$ Our study was contradictory to a pilot study done to see the effect and toxicity of atorvastatin on normal male patients for a total period of 5 months, which concluded saying, atorvastatin caused a decrease in sperm function and its parameters when used for long periods of time. ${ }^{29}$

The mechanism by which atorvastatin causes an increase in sperm parameters and helps in reversing fertility changes caused due to stress can be attributed to its antioxidant action, a known pleiotropic effect which is the current topic of research globally. In an interventional trial conducted in Cleveland clinic, among non-coronary vascular disease patients with an light density lipoprotein (LDL) level above $130 \mathrm{mg} / \mathrm{dL}$, it was found that 
atorvastatin helped in reducing the specific molecular footprints of distinctive oxidative pathways with a specific decline in markers responsible for myeloperoxidase-derived and nitric oxide-derived oxidants in blood vessels. This action was independent of the changes induced by statins on lipid parameters and C-reactive protein (CRP). ${ }^{19}$

\section{ConcLusion}

In conclusion, the rats subjected to the forced swim test had decreased bodyweight, testicular weight, sperm count, sperm motility, and sperm viability. Both vitamin $C$ and atorvastatin either alone or in combination have protected against the harmful effect of stress on sperm parameters significantly. The combination of drugs is not superior to either of the drugs alone.

\section{Clinical Significance}

Further preclinical research is also needed to compare the effects of other drugs in a class of statins and to find out whether all drugs belonging to statins cause a similar or different effect. As atorvastatin is comparable to vitamin $C$ as an antioxidant in our study, it adds to the research in the field of medicine by throwing light on the pleiotropic effects of statins apart from its lipid-lowering effect.

\section{Presentation}

Best research paper award at IFFS 2016, world congress of infertility, and won cash prize of 3000 rupees and complete waiver of registration fees.

\section{Acknowledgment}

The authors would like to thank Dr K. L Bairy for his mentorship and Department of Pharmacology for the help.

\section{References}

1. Zegers-Hochschild F, Adamson GD, et al. International Committee for Monitoring Assisted Reproductive Technology, World Health Organization. Fertil Steril 2009 Nov;92(5):1520-1524.

2. Calverton, Maryland, USA: ORC Macro and the World Health Organization; 2004. World Health Organization. Infecundity, Infertility, and Childlessness in Developing Countries. DHS Comparative Reports No 9.

3. Sciarra J. Infertility: an international health problem. Int J Gynaecol Obstet 1994 Aug;46(2):155-163. DOI: 10.1016/0020-7292(94) 90230-5.

4. Population Council. Infertility. Looking Back, Looking Forward: A Profile of Sexual and Reproductive Health in India. New Delhi: Population Council, 2004; pp. 67-72.

5. Sadock BJ, Sadock VA. Kaplans and Sadocks Symptoms of Psychiatry Behavioral Sciences Clinical Psychiatry, 9th ed. Philadelphia: Lippincott Williams and Wilkins; 2003. pp. 872-874.

6. World Health Organization. WHO Laboratory Manual for the Examination of Human Semen and Semen-Cervical Mucus Interaction, 4th ed. Cambridge: Cambridge University Press; 1999. pp. 1-86.

7. Men's Health - Male Factor Infertility. University of Utah Health Sciences Center. 04 January, 2003. Archived from the Original on 04 July 2007. [Last retrieved on 2007 Nov 11]. Available from: http:// web.archive.org/web/20080620064743/http://healthcare.utah.edu/ healthinfo/adult/men/infertil.htm.
8. Brugh 3rd VM, Lipshultz LI. Male factor infertility: Evaluation and management. Med Clin North Am 2004;88:367-385. DOI: 10.1016/ S0025-7125(03)00150-0.

9. Hirsh A. Male subfertility. BMJ 2003 Sep 20;327(7416):669-672. DOI: 10.1136/bmj.327.7416.669.

10. Lotti F, Maggi M. Ultrasound of the male genital tract in relation to male reproductive health. Hum Reprod Update 2015;21:56-83. DOI: 10.1093/humupd/dmu042.

11. Montoro J, Mullol J, et al. Stress and allergy. J Investig Allergol Clin Immunol 2009;19(1):40-47.

12. Stefan $\mathrm{S}$, Anthony $\mathrm{K}$, et al. Is there a link between exercise and male factor infertility? Open Reprod Sci J 2011;3:105-113. DOI: 10.2174/1874255601103010105.

13. Prasad Vijay S, Ghongane BB, et al. An experimental study on effect of antioxidant vitamin $\mathrm{E}$ in stress and alcohol induced changes in male fertility in albino rats. Int J Med Res Health Sci 2012;1(1):1-6.

14. Yoon $\mathrm{H}$, Chung WS, et al. Effects of stress on female rat sexual function. Int J Impot Res 2005;17:33-38. DOI: 10.1038/sj.ijir.3901223.

15. Aitken RJ, Roman SD. Antioxidant systems and oxidative stress in the testes. Oxid Med Cell Longev 2008;1(1):15-24. DOI: 10.4161/ oxim.1.1.6843.

16. Nayanatara AK, Vinodini NA, et al. Role of ascorbic acid in monosodium glutamate mediated effect on testicular weight, sperm morphology and sperm count, in rat testis. J Chin Clin Med 2008;3(1):1-5. DOI: 10.1186/1749-8546-3-1.

17. Rekha DK, Nayanatara AK, et al. Infertility in male Wistar rats induced by cadmium chloride: role of ascorbic acid. J Chin Clin Med 2009;4(11):616-621.

18. Vijayprasad $S, B b$ G, et al. Effect of Vitamin C on Male Fertility in Rats Subjected to Forced Swimming Stress. J Clin Diagn Res 2014;8(7):HC05-HC08. DOI: 10.7860/JCDR/2014/8432.4622.

19. Shishehbor $M H, B r e n n a n M L$, et al. Statins promote potent systemic antioxidant effects through specific inflammatory pathways. Circulation 2003 Jul 29;108(4):426-431. DOI: 10.1161/01. CIR.0000080895.05158.8B.

20. Porsolt RD, LePichon $M$, et al. A new animal model sensitive to antidepressant treatments. Nature 1977;266:730-732. DOI: 10.1038/ 266730a0.

21. Nayanatara AK, Nagaraja HS, et al. The effect of repeated swimming stress on organ weights and lipid peroxidation in rats. Thai Journal of Physiological Sciences 2005;18(1):3-9.

22. Search FL, Samuel BO, et al. Testicular toxicity and sperm quality following cadmium exposure in rats: Ameliorative potentials of Allium cepa. J Hum Reprod Sci 2012;5(1):37-42. DOI: 10.4103/09741208.97798.

23. Saalu LC, Kpela T, et al. The Dose-Dependent Testiculoprotective and Testiculotoxic Potentials of Telfairia occidentalis Hook F. Leaves Extract in Rat. Int J Appl Res Nat Prod 2010;3(3):27-38.

24. Metwally $S A$, Hekma $A E$, et al. The protective effect of Linseed against carbendazim induced testicular toxicity in rats. Eur J Sci Res 2011;49(2):208-224.

25. Raj J, Pandey S, et al. Testosterone hormone level in albibo rats following restraint stress of long duration. J Ana Soc India 2004;53(1):17-19.

26. Nashwa A, Abu A, et al. The protective effect of Ginger and N-Acetyl cystine on ciprofloxacin induced reproductive toxicity in male rats. Am J Sci 2011;7(7):741-752.

27. Paolicchi A, Pezzini A, et al. Localization of a GSH-dependent dehydroascorbate reductase in rat tissues and subcellular fractions. Arch Biochem Biophys 1996;333:489-495. DOI: 10.1006/ abbi.1996.0419.

28. Shalaby MA, El Zorba HY, et al. Effect of a-tocopherol and simvastatin on male fertility in hypercholesterolemic rats. Pharmacol Res 2004 Aug 31;50(2):137-142. DOI: 10.1016/j.phrs.2003.10.013.

29. Pons-Rejraji $\mathrm{H}$, Brugnon $\mathrm{F}$, et al. Evaluation of atorvastatin efficacy and toxicity on spermatozoa, accessory glands and gonadal hormones of healthy men: a pilot prospective clinical trial. Reprod Biol Endocrinol 2014;12:65. DOI: 10.1186/1477-7827-12-65. 